\title{
Understanding Epilepsy among Parents Through an Educative Blog: A Novel Method
}

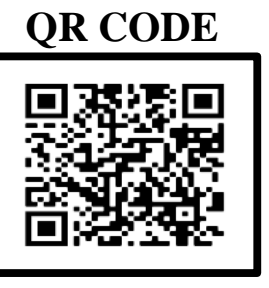

\section{KAVYA KG ${ }^{* 1}$, SHRUTHI B. PATIL ${ }^{2}$}

INTRODUCTION: Epilepsy is a neurological disorder characterized by the risk of recurrent seizures. Individuals are still socially discriminated due to misunderstandings by widespread negative attitudes and defensive behavior.

AIM: To assess the knowledge among parents regarding epilepsy and to eradicate the myths associated with using a blog.

MATERIALS AND METHOD: A blog was created with elaborate information regarding Epilepsy.50 parents of children with history of Epilepsy

were included for the study. The parents of the children were given the link to the blog and were asked to go through it and express their views and responses. Later the parents were educated and doubts/queries were clarified.

RESULTS: About 40 parents were willing to go through the blog. The other 10 though reluctant went through the blog after making them realize the need to have knowledge about epilepsy. Among 50 parents, around 30 parents refused to believe that it was a form of epilepsy/fits and discontinued the medication without Physician's consent. Around 45 parents believed that giving metal objects helped in treating seizures. Also 40 parents believed strongly that Febrile seizures was not a form of seizures and did not require treatment. 20 parents had discontinued the medication on their own after a few months.

CONCLUSION: In our study we found lack of knowledge regarding Epilepsy and the different variants of epilepsy. Also, parents strongly believed in the myths and hence did not consult a doctor. Most parents assumed Epilepsy to be a neurologic disorder and that it required psychiatric help. This study made an attempt to educate people regarding epilepsy in detail by the means of an educative blog.

KEYWORDS: Epilepsy, Social Discrimination, Seizures, Blog

\section{INTRODUCTION}

Epilepsy is a neurological disorder characterized by the risk of recurrent seizures. Epileptic seizures may vary from brief and undetectable seizures to long periods of vigorous shaking. In a developed country, around 45/100o persons have epilepsy. The risk of epilepsy increases with increase in age. In developing countries, this rate can be as high as 43 per 1,0oo people. ${ }^{1-5}$

According to the International League Against Epilepsy, epilepsy is diagnosed when a person has 2 or more unprovoked seizures. A seizure is classified as "partial" when the electrical discharge causing it occurs in a specific area of the brain or "generalized" when the discharge affects the entire brain cortex. When there is loss of awareness, seizures are termed complex. The classification of epilepsy is similar. Epilepsy can be partial or generalized. Based on the cause, it can be symptomatic (caused by a developmental malformation), idiopathic (when a genetic condition is responsible) or cryptogenic/idiopathic (when the cause is unknown). ${ }^{6}$

Social acceptance of people with epilepsy largely depends on the ideas of people in the society and is often a considerable problem for the patients and their families. Individuals are still socially discriminated due to misunderstandings by widespread negative attitudes and defensive behavior. This can affect people economically, socially, and culturally. In India and China, epilepsy is used as a justification to deny marriage. ${ }^{2}$ People in some region believe that epilepsy is a demonic possession. People in Tanzania and few parts of Africa think epilepsy is associated with evil spirit, witchcraft, or poisoning, and it is believed to be contagious, for which there is no evidence. In the United Kingdom, before the $197 \mathrm{o}_{\mathrm{s}}$, there were laws that prevented epileptic patients from getting married. It also brings about a feeling of shame among the patients that most of them deny to accept they have a seizure. ${ }^{-}$ 13

Negative attitude towards patients with epilepsy by the family, friends, relatives, school mates and also teachers affects the morale of the patient and the parents to a large extent. Literature search showed many questionnaire studies published regarding epilepsy but there was paucity of studies which imparted knowledge to people regarding epilepsy. The different myths associated and the perceptions of different people towards epilepsy by using technological aids. Hence this study aimed to educate people regarding epilepsy in detail by the means of an educative blog. 


\section{MATERIALS AND METHOD}

A blog was created with elaborate information comprising the definition of Epilepsy, types, common myths associated, signs and symptoms, perceptions of family, relatives, batchmates and school teachers regarding epilepsy and the investigations. This is the link to the bloghttps://kaviekg.blogspot.com/2019/o6/epilepsy.html?m $=1$. The patients with history of Epilepsy visiting the Department of Special Health Care needs, SDM Dental college, a constituent unit of Shri Dharmasthala Manjunatheswara University, Dharwad were included for the study.

In the first visit the parents were given the link and were asked to go through the blog and give their comments below. The parents were also told to clarify any queries they had in this regard. Total of 50 children with history of Epilepsy were taken as study subjects.

A total of 50 children with history of Epilepsy were included in the study. Among these it was seen that 30 children had history of febrile seizures who continued medication with anti-epileptic medication thereafter. The other 20 children gave history of Tonic Clonic seizures and Static seizures. Only 20 children were on medication and visited the physician frequently.

\section{RESULTS}

About 40 parents were willing to go through the blog. The other 10 though reluctant went through the blog after making them realize the need to have knowledge about epilepsy. Among 50 parents, around 30 parents refused to believe that it was a form of epilepsy/fits and discontinued the medication without Physician's consent. 10 parents had basic knowledge, they were aware of the attack and the need for medication but they believed in the myths.10 patients had thorough knowledge about epilepsy and visited the physician regularly (Table 1 )

Around 45 parents believed that giving metal objects helped in treating seizures. Also 40 parents believed strongly that Febrile seizures was not a form of seizures and did not require treatment. 20 parents had discontinued the medication on their own after a few months. 35 parents gave history of epilepsy without frothing but only jerky hand movements and eyes staring into space. 35 parents did not consult a physician and 15 parents consulted a physician. 40 parents agreed that it was a mental illness and 10 parents did not agree. 40 parents believed that it was a mental illness and to parents did not believe so (Figure 1).

35 parents believed seizures could be cured by priest whereas 15 did not believe so. 30 children felt that they were isolated in school whereas 20 did not feel so. 40 parents believed it was a taboo in society and they were considered as bad omen whereas 10 did not consider. Only 15 parents had got the investigation like EEG done for seizures whereas 35 patients had not undergone investigations. 40 parents said they benefitted from the blog because they had no information regarding epilepsy in depth. ${ }^{10}$ parents were not very keen on listening as few already had information and few were very rigid and stuck on to those myths (Figure 2).

\section{DISCUSSION}

Epilepsy has been long recognized as a stigma in society and a lot of misconceptions are present among the common public regarding Epilepsy. Since many years people believe in these myths and so deep rooted it is a herculean task to uproot these beliefs from the minds of the public.

There are myths which are believed to be true and are the major cause for the social stigma regarding Epilepsy. Some cultures believe that epilepsy represents demonic possession. Although epilepsy arises from a transient dysfunction in the brain, fear and ignorance still lead to discrimination and feelings of shame. In the laws of some countries, epilepsy is strongly associated with mental illness and cognitive disabilitiesunfortunate generalizations that unfairly affect many people with epilepsy. Such pervasive social stereotyping is difficult to overcome. ${ }^{6}$

In our study we found lack of knowledge regarding Epilepsy and the different variants of epilepsy. Also, people strongly believed in the myths and hence did not consult a doctor.

Most parents assumed Epilepsy to be a neurologic disorder and that it required psychiatric help. It was also noticed that the parents strongly believed that it could be cured by a priest or Shaman (person possessing divine power) and hence consulting a doctor was unnecessary. Parents also agreed that relatives and other schoolmates including the teachers treated the children differently as compared to other children. Another common finding was that parents discontinued the medication soon after the symptoms were reduced. 


\begin{tabular}{|c|c|c|c|}
\hline S NO. & QUESTION & YES & NO \\
\hline 1 & Epilepsy is a mental illness & 40 & 10 \\
\hline 2 & History of epilepsy without frothing & 35 & 15 \\
\hline 3 & Febrile seizures is not a form of seizures & 40 & 10 \\
\hline 4 & Parents consulted a physician & 15 & 35 \\
\hline 5 & Giving metal objects helped in treating seizures & 45 & 5 \\
\hline 6 & Seizures can be cured by priest & 35 & 15 \\
\hline 7 & Discontinued the medication on their own & 20 & 30 \\
\hline 8 & Parents hesitant to reveal condition & 20 & 30 \\
\hline 9 & Taboo in society and bad omen & 40 & 10 \\
\hline 10 & Parents of children with epilepsy were not invited to family events & 20 & 30 \\
\hline 11 & Relatives spoke ill about these children and their parents & 25 & 25 \\
\hline 12 & Other parents did not let their children mingle with these children & 30 & 20 \\
\hline 13 & School teachers looked down upon them & 10 & 40 \\
\hline 14 & Children felt that they were isolated in school & 30 & 20 \\
\hline 15 & Investigations like EEG & 15 & 35 \\
\hline 16 & Benefitted from the blog & 40 & 10 \\
\hline
\end{tabular}

Table 1. List of Questions Asked to Parents

Parents of patients with Febrile epilepsy believed that it was not a form of epilepsy and just a manifestation of high fever. Parents who gave history of one attack described symptoms of jerky movements of hands and legs, eyes staring into space which was occasionally accompanied by frothing. After reading the blog, the parents revealed that it was ignorance which made that

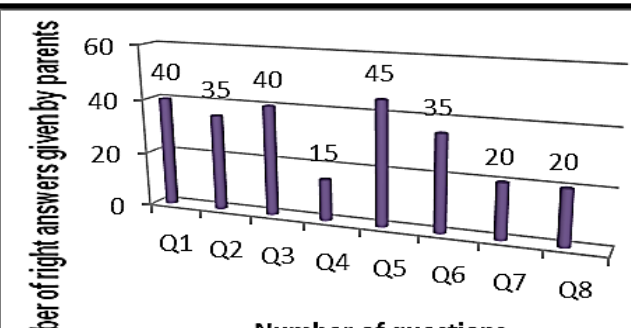

Number of questions

Figure 1. Graphical Representation of the Appropriate Responses Provided by Parents for the Blog Questionnaire (Q1-Q8) them believe in the myths and also the fear and they were educated in depth regarding Epilepsy.

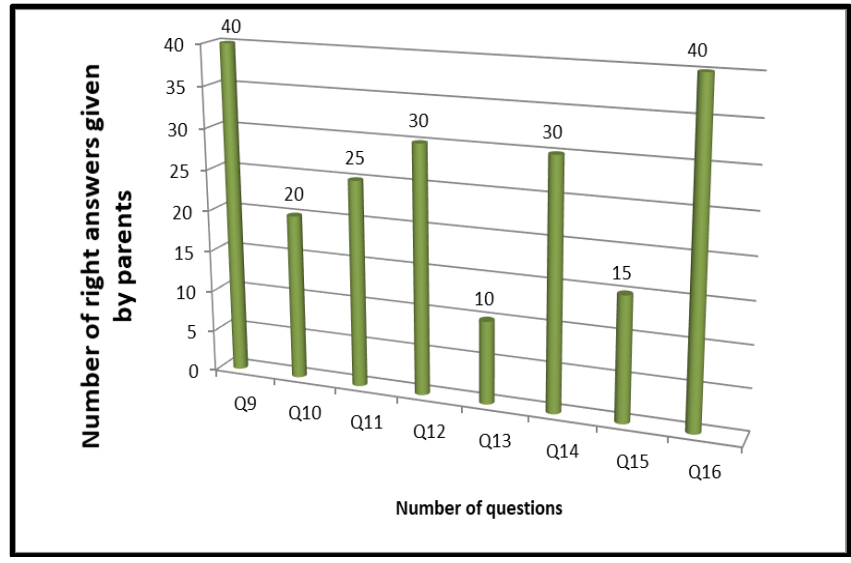

Figure 2. Graphical Representation of the Appropriate Responses Provided by Parents for the Blog Questionnaire (Q9-Q16) 


\section{CONCLUSION}

The social stigma surrounding Epilepsy cannot be eradicated overnight but by small efforts we sure can bring about a change in the mindset of the people regarding Epilepsy. As it is the era of social media, we can use it as a means of educating the people and trying to remove all these superstitious beliefs from the minds of people to let the child live to its best. This is the first of its kind study wherein a blog was formulated to educate people regarding epilepsy.

\section{REFERENCES}

1. Fisher RS, Acevedo C, Arzimanoglou A, Bogacz A, Cross JH, Elger CE, et al. ILAE official report: A practical clinical definition of epilepsy. Epilepsia 2014;55:475-82.

2. Chaudhari VK, Kumar P, Yadav V, Pathak D, Hussain Z. A brief overview of epilepsy. Glob J Med Res A: Neurol Nerv Syst. 2016;16(1-A):1-6.

3. Scott RA, Lhatoo SD, Sander JW. The treatment of epilepsy in developing countries: Where do we go from here? Bull World Health Organ 2001;79:344-51.

4. Brodie MJ, Elder AT, Kwan P. Epilepsy in later life. Lancet Neurol. 2009;8:1019-30.
5. Aragon CE, Burneo JG.Understanding the Patient with Epilepsy and Seizures in the Dental Practice JCDA. February 2007;43(1):71-6.

6. Aragon CE, Hess T, Burneo JG, Knowledge and Attitudes about Epilepsy: A Survey of Dentists in London, Ontario JCDA .July/August 2009;75(6):4504509 .

7. de Boer HM. Epilepsy stigma: Moving from a global problem to global solutions. Seizure 2010;19:630-6.

8. Burneo JG. Sonko-nanay and epilepsy among the Incas. Epilepsy Behav 2003;4:181-4.

9. Jilek-Aall L. Morbus sacer in Africa: Some religious aspects of epilepsy in traditional cultures. Epilepsia 1999;40:382-6.

10. Newton CR, Garcia HH. Epilepsy in poor regions of the world. Lancet 2012;380:1193-201.

11. Baker GA, Brooks J, Buck D, Jacoby A. The stigma of epilepsy: A European perspective. Epilepsia 2000;41:98104.

12. Paschal AM, Ablah E, Wetta-Hall R, Molgaard CA, Liow K. Stigma and safe havens: A medical sociological perspective on African-American female epilepsy patients. Epilepsy Behav 2005;7:106-15.

13. Jacoby A, Snape D, Baker GA. Epilepsy and social identity: The stigma of a chronic neurological disorder.

Lancet Neurol 2005;4:171-8.

Source of support: Nil, Conflict of interest: None declared 